\title{
Charm and strange hadron spectra from overlap fermions on HISQ gauge configurations
}

\section{S. Basak ${ }^{a}$, S. Datta ${ }^{b}$ Padmanath M. $^{b}$, P. Majumdar ${ }^{c}$, and N. Mathur ${ }^{* b}$ \\ (Indian Lattice Gauge Theory Initiative)}

${ }^{a}$ School of Physical Sciences, National Institute of Science Education and Research, Bhubaneswar 751 005, India

${ }^{b}$ Department of Theoretical Physics, Tata Institute of Fundamental Research, Homi Bhabha Road, Mumbai 400005, India

${ }^{c}$ Department of Theoretical Physics, Indian Association for the Cultivation of Science, Kolkata 700032, India.

E-mail: nilmaniatheory.tifr.res.in

We report here results on charm and strange hadron spectra. Adopting a mixed action approach, we use overlap fermions for valence quarks, on a background of $2+1+1$ flavours HISQ gauge configurations of MILC collaboration. Two lattice spacings $(0.09 \mathrm{fm}$ and $0.06 \mathrm{fm})$ are used. We find the hyperfine splitting of $1 \mathrm{~S}$ charmonia to be $114_{-2}^{+3} \mathrm{MeV}$ and $109_{-3}^{+4} \mathrm{MeV}$, and the splitting $\left(m_{\Omega_{c c c}}-\frac{3}{2} m_{J / \Psi}\right)$ is found to be $110_{-10}^{+20} \mathrm{MeV}$ and $120(10) \mathrm{MeV}$, corresponding to lattices with spacings $a=0.09$ and $0.06 \mathrm{fm}$ respectively. We also look at the ratio of the leptonic decay constant $f_{D_{s}^{*}} / f_{D_{s}}$.

The 30 International Symposium on Lattice Field Theory - Lattice 2012,

June 24-29, 2012

Cairns, Australia

*Speaker. 


\section{Introduction}

During the last few years MILC lattice collaboration has generated [1] a large set of configurations with the one-loop, tadpole improved Symanzik gauge action and the highly improved staggered quark (HISQ) fermion action [2]. Four flavours of dynamical sea quarks: degenerate up and down, strange, and charm, were included in these configurations. Taste violations in the HISQ action were found to be small [2]. On the other hand, overlap fermion action [3] has exact chiral symmetry $[3,4]$ on the lattice and is automatically $\mathscr{O}(a)$ improved. It has also many desirable features, such as the adaptation of multi mass algorithms [5]. However, using overlap action for the dynamical quarks is still prohibitively costly, except with fixed topology [6]. In this work we have adopted a mixed action formalism where we used overlap valence quarks on the HISQ gauge configurations generated by MILC collaboration [1]. By adopting such a mixed action approach, one can get advantage of the chiral symmetry and low quark mass limit of overlap fermions, and the advantage of having a large set of configurations with small discretization errors as well as small taste breaking effects. One also gets the advantage of simulating both light, strange as well as heavy fermions on the same lattice formalism with chiral fermions having no $\mathscr{O}(a)$ errors. Of course we will have to encounter the usual issues related to mixed action and partial quenching [14]. Similar approach has been taken by the $\chi Q C D$ collaboration, who used overlap valence quarks on $2+1$ flavours dynamical domain wall gauge configurations [7].

In this work, by using above mentioned mixed action approach, we report our preliminary results on charm and strange hadron spectra as well as leptonic decay constants for $D_{s}$ and $D_{s}^{*}$ mesons.

\section{Numerical details}

We used two sets of dynamical 2+1+1 flavours HISQ lattice ensembles, generated by the MILC collaboration : a set of $32^{3} \times 96$ lattices with lattice spacing $a=0.09 \mathrm{fm}$, and a set of $48^{3} \times 144$ lattices with $a=0.06 \mathrm{fm}$. The details of these configurations are summarized in ref. [1]. The results reported here were obtained from 100 configurations on the coarser lattice, and 35 configurations on the finer lattice. Here we did not address the uncertainties in the determination of lattice spacings which we will address in subsequent works.

For valence quarks we used overlap action [3]. For the numerical implementation of massive overlap fermions the methods used by the $\chi Q C D$ collaboration [8] were followed. To evaluate the sign function, the Zolotarev approximation is used, and the low Wilson eigenmodes are projected out by Arnoldi method. We used 350 and 160 Wilson eigenmodes for the coarser and the finer lattices respectively. The constraint here came from the size of the memory of the machine. Using multimass CG algorithm we were able to input a range of quark masses covering from light, strange to heavy quarks. It is worthwhile to mention that one should not use the same precision for the evaluation of light and heavy quark propagators. While light quark and strange quark propagators needs relatively lower precision, for heavy quark propagators which decay rapidly, having higher precision in the inversion is absolutely necessary. Otherwise, one will get flat hadron correlators at the middle of the lattice. However, for using different precisions in the multimass we found that it is not necessary to reload the eigenmodes repeatedly, which increases the total inversion time. We used periodic boundary condition in the spatial and antiperiodic in the temporal directions. 
Gauge configurations were first fixed to coulomb gauge and then HYP smeared (one HYP). Using both point and wall sources we calculated various point-point, wall-point as well as wall-wall correlators.

The strange mass was tuned by setting the $\bar{s} s$ pseudoscalar mass to $685 \mathrm{MeV}$ [9]. With the strange mass tuned this way, the mass of the vector $\phi(\bar{s} s)$ meson was found to be $1050(20) \mathrm{MeV}$ and 1030(10) $\mathrm{MeV}$ on the coarser and finer lattices respectively, and the mass of the $\Omega$ (sss) baryon was found to be 1620(40) and $1630(30) \mathrm{MeV}$ respectively. The charm mass is tuned by setting the spin-averaged $1 \mathrm{~S}$ state mass, $\left(m_{\eta_{c}}+3 m_{J / \psi}\right) / 4$, to its physical value. The charm quark mass $\left(m a_{\text {charm }}\right)$ in these lattices are obtained to be 0.55 and 0.336 , respectively. Interestingly, the $\overline{M S}$ mass of charm, $1.275 \mathrm{GeV}$, corresponds to $m a=0.582$ and 0.388 , respectively, indicating that the mass renormalization constant $Z_{m}^{\overline{M S}}$ is close to 1 for this action.

Since $m a$ is not very small, we need to be careful about discretization errors. Overlap action does not have $O(\mathrm{ma})$ errors. In order to estimate the size of discretization errors coming from higher orders of $m a$, we look at the energy-momentum dispersion relation of the $1 \mathrm{~S}$ charmonia. We calculated the mesons at various external momenta $p^{2}=(2 \pi / L)^{2} n^{2}$, with $n \leq 2$. We used both point source and a momentum-induced wall source for the finite momenta mesons. For the wall source at finite momenta, we put a suitable phase factor in the wall so as to project to the suitable momentum. While this requires a separate inversion for each momenta, that is more than compensated by the improvement in the signal, as shown in Fig. 1. In both figures we show both point source and wall source results for the coarser lattice. Note that the wall source results are from 8 configurations, but the statistical error is already comparable to that from 100 configurations with the point source.

In the left panel of Fig. 1, we show $E(p)$ for various momenta for the pseudoscalar meson on our coarser lattices. Also shown are the continuum dispersion relation, $E^{2}=m^{2}+p^{2}$, and the lattice dispersion relation for the standard scalar action with $O(m a)^{2}$ error, $\sinh ^{2}(E(p) a / 2)=$ $\left(\sinh ^{2}(m a / 2)\right)^{2}+\sin ^{2}(p a / 2)$. While substantial deviation from the continuum dispersion relation is seen, the lattice scalar dispersion relation seems to explain the data quite well. For a more quantitative analysis, we follow the standard practice of introducing an effective "speed-of-light" $c$ through $E^{2}(p)=m^{2}+p^{2} c^{2}$. The value of $c$ obtained using this relation and $E(p)$ at various $p$ are shown in the right panel of Fig. 1. As the figure shows, we get $c^{2} \sim 0.75$ on our coarser lattices, and $c^{2} \sim 0.89$ on the finer lattices. Using quenched overlap fermions, on an even coarser lattice, ref. [10] found $c^{2} \sim 1$ for the charm. However, on our dynamical lattices, we obtained $c^{2}$ values similar to that obtained in the literature with clover action, indicating a similar size of cutoff in the overlap action as that in the clover. The $c^{2}$ values are well-approximated by the estimate $m a / \sinh (m a)$, suggested by the lattice scalar action.

\section{Results}

We utilized both point and wall sources to generate a large set of quark propagators. Using those we calculated various hadron correlators and extracted light, strange and charm hadron masses. The pseudoscalar meson masses ranged between $240-3582 \mathrm{MeV}$ for the coarser lattices and between $420-5275 \mathrm{MeV}$ for the finer lattices. In Fig. 2 we plot the pseudoscalar meson masses over a range of quark masses. 

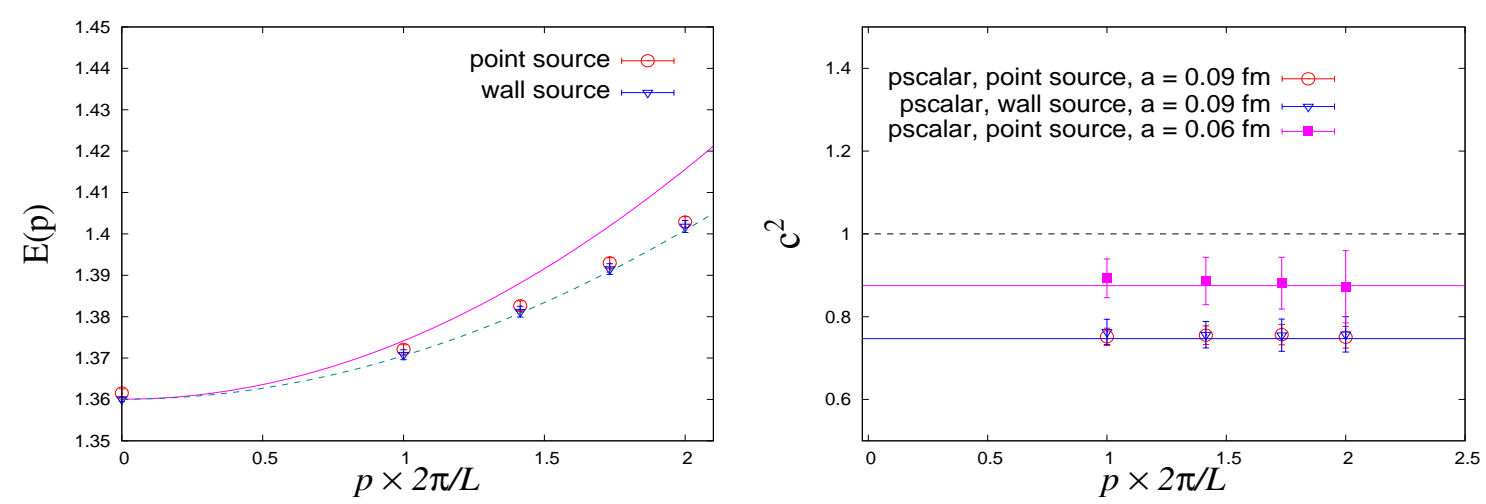

Figure 1: (Left) Energy-momentum dispersion relation for the $\eta_{c}$ on the lattices with $a=0.09$ fm. Also shown are the continuum relativistic dispersion relation (solid line), and the lattice dispersion relation (dashed line) for the standard scalar action. (Right) $c^{2}=\left(E^{2}(p)-E^{2}(p=0)\right) / p^{2}$ calculated for $\eta_{c}$ at various values of $p$, on lattices with $a=0.09$ and $0.06 \mathrm{fm}$. Solid horizontal lines represent the estimate $\mathrm{ma} / \sinh (\mathrm{ma})$, suggested by the lattice scalar action.

Using these correlators, we report here preliminary results for spectra of charmonia, mesons of $D_{s}$ family, and charmed baryons. We also report preliminary studies of leptonic decay constants of $D_{s}$ and $D_{s}^{*}$ mesons.
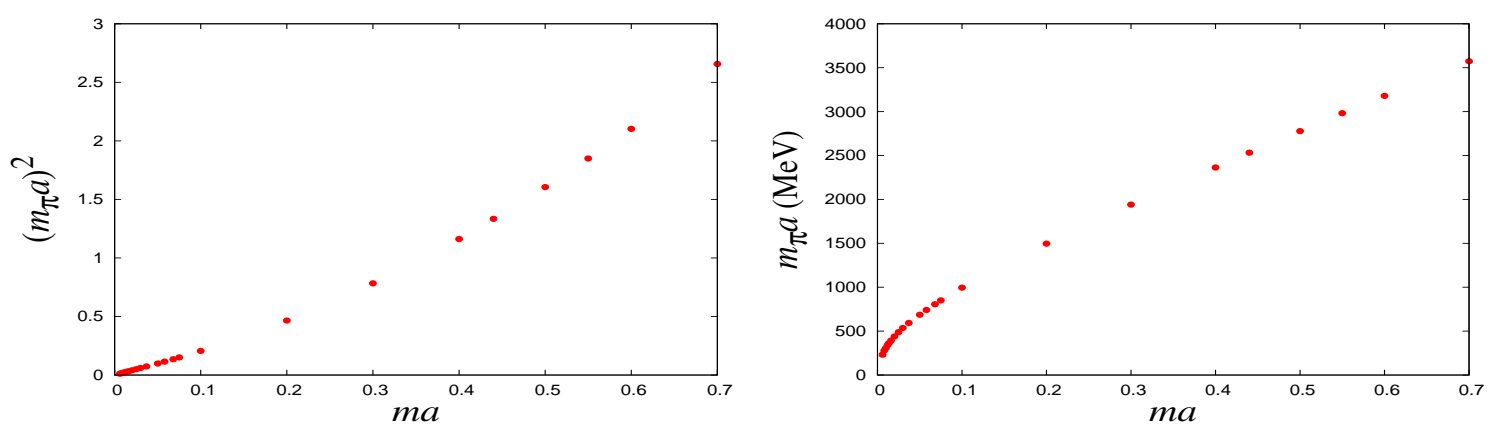

Figure 2: Pseudoscalar meson masses over a range of light to heavy quark masses. Left figure is for $m_{\pi}^{2} a$ as function of quark mass $m a$, and the right figure is for $m_{\pi} a$ vs $m a$ in physical unit.

\subsection{Hyperfine splitting in 1S charmonia}

The hyperfine splitting in 1S charmonia is one of the most well studied physical quantities in lattice charmonium calculations over the year, and until very recently [11] lattice results were found to be smaller than the experimental value $(\sim 116 \mathrm{MeV})$. This underestimation was mainly due to the discretization error associated with the charm quark action and the quenched approximation. In our study we also calculated this splitting. In Fig. 3, we showed the effective splittings between vector and pseudoscalar correlators (jackknifed) at the tuned charm mass for wall-point correlators. Horizontal lines shown are the fit results obtained by fitting only these correlators. Our final estimated results, by simultaneously fitting the wall-point and point-point correlators, for this hyperfine splitting are $114_{-2}^{+3} \mathrm{MeV}$ and $109_{-3}^{+4} \mathrm{MeV}$ corresponding to lattices with spacings $a=$ 0.09 and $0.06 \mathrm{fm}$ respectively. 

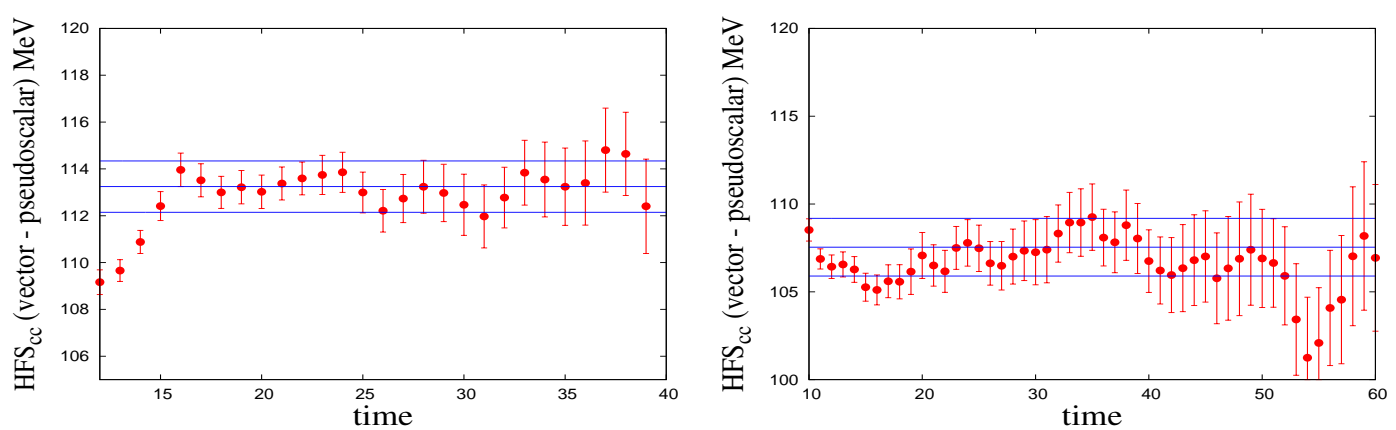

Figure 3: Effective hyperfine splitting in $1 \mathrm{~S}$ charmonia for wall-point correlators for lattices with spacing 0.09 (left) and $0.06 \mathrm{fm}$ (right). Horizontal lines show the fit results obtained by fitting only these correlators.

\subsection{Charm and strange mesons}

Along with pseudoscalar and vector mesons we also calculated the ground state spectra of tensor, axial and scalar charmonia. Besides charmonia we also calculated the strange-charm $D_{s}$ mesons. In Fig. 4(a) we showed splittings between these various mesons at two lattice spacings. It is to be noted that the splitting of $D_{s}-\eta_{c} / 2$, which has smaller lattice spacing error [12], obtained is consistent with its experimental value.

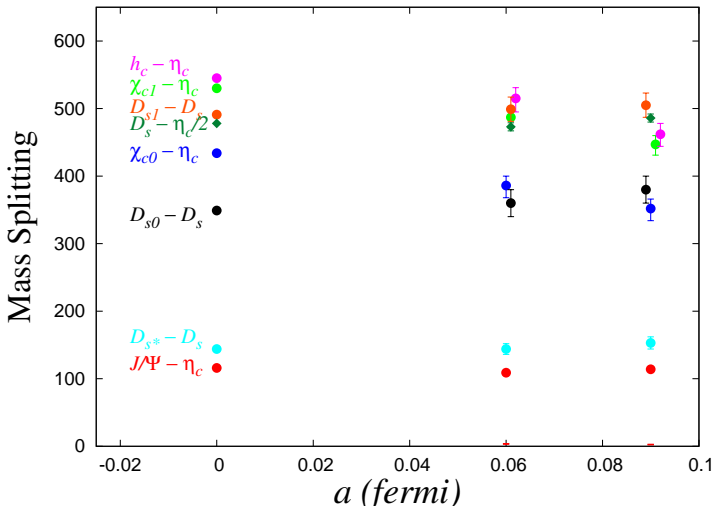

Figure 4: (a) Meson mass splitting for charmonia and strange-charm mesons at two lattice spacings. Ex-

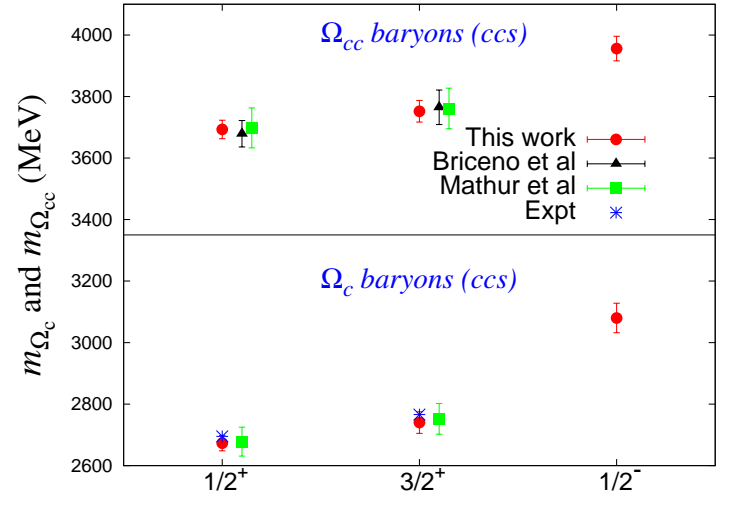

Figure 4: (a) Meson mass splitting for charmonia and strange-charm mesons at two lattice spacings. Experimental values are shown in the left side. (b) $\Omega_{c}$ and $\Omega_{c c}$ baryon masses. Also shown are other lattice determinations, and the experimental values, where available.

\subsection{Charmed baryons}

Recently, there have been exciting developments in heavy-baryon physics, both theoretically and experimentally. Results from LHC and future charm-bottom factories are expected to add to the excitement in this field in the near future. On our lattices we extracted ground state spectra of charmed baryons with one or more charm quark content, for example, baryons with quark content csu,cuu,css and ccs. In fig. 4(b) we showed results for $\Omega_{c}(c s s)$ and $\Omega_{c c}(c c s)$ baryons. It is to be noted that for these baryons we extracted masses for both spin $1 / 2$ and spin $3 / 2$ with both parities, some of which are yet to be measured experimentally. Our results are consistent with other lattice results [13], and the experimental values, where available. Results for other charm baryons at various pion masses were shown in Fig. 5. The chiral extrapolation will involve effects from partial quenching as well as mixed action formalism. Mixed action partially quenched chiral perturbation theory (MAPQ $\chi \mathrm{PT}$ ) has been developed for various mixed action formalisms [14] 
which requires calculation of $\Delta_{\text {mix }}$ [15], the low energy constant representing $\mathscr{O}\left(a^{2}\right)$ discretization dependence. In future, utilizing MAPQ $\chi \mathrm{PT}$ we will extrapolate these masses to the physical limit. We also calculated the experimentally unknown triply charmed baryon $\Omega_{c c c}$, and a better way to quote its mass is through the splitting of $m_{\Omega_{c c c}}-\frac{3}{2} m_{J / \Psi}$, which we extracted to be $110_{-10}^{+20} \mathrm{MeV}$ and $120(10) \mathrm{MeV}$, for $0.09 \mathrm{fm}$ and $0.06 \mathrm{fm}$ lattices respectively.
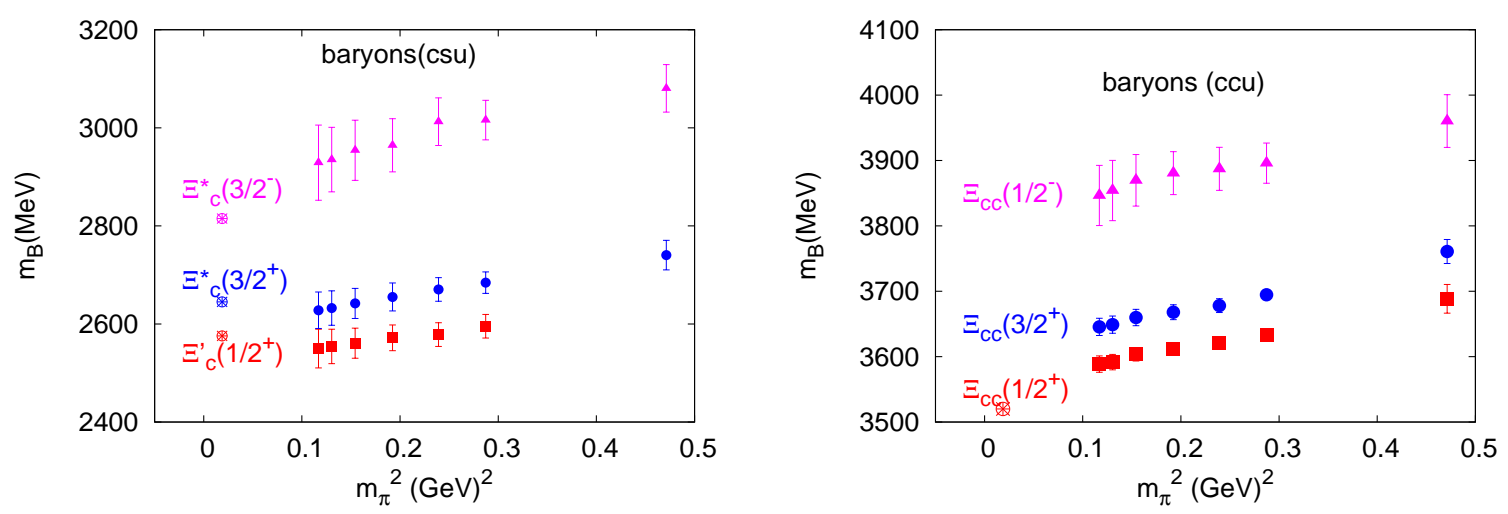

Figure 5: Ground state spectra for charmed baryons at various pion masses for $32^{3} \times 96, a=0.09 \mathrm{fm}$ lattice.

\subsection{Decay constants}

The decay constants of heavy-light mesons are experimentally very important: they are essential ingredients in extracting CKM matrix elements from decays of heavy-light mesons. For the suitable continuum currents $\left\{A_{\mu}, V_{\mu}\right\}=\left\{Z_{A}\left(\bar{s} \gamma_{\mu} \gamma_{5} c\right)_{\text {lat }}, Z_{V}\left(\bar{s} \gamma_{\mu} c\right)_{\text {lat }}\right\}$, where $Z_{A}, Z_{V}$ are the renormalization constants to match the lattice operators to continuum currents, the leptonic decay constants for the pseudoscalar $D_{s}$ and the vector $D_{s}^{*}$ are defined as

$$
\left\langle 0\left|A_{\mu}\right| D_{S}(p)\right\rangle=i f_{D_{s}} p_{\mu}, \quad\left\langle 0\left|V_{\mu}\right| D_{s}^{*}(p, \lambda)\right\rangle=i f_{D_{s}^{*}} m_{D_{s}^{*}} \varepsilon_{\mu}^{\lambda},
$$

where $\varepsilon_{\mu}^{\lambda}$ is the polarization vector for the vector meson. From the amplitude of the $\langle V(t) V(0)\rangle$ correlator, $f_{D_{s}^{*}}$ can therefore be extracted using standard techniques. Similarly, $f_{D_{s}}$ can be extracted from $\left\langle A_{4}(t) P(0)\right\rangle$ and $\langle P(t) P(0)\rangle$ correlators, where $P=Z_{P} \bar{s} \gamma_{5} c$ is the pseudoscalar current.

Employing point-point as well as wall-point and wall-wall correlators we extracted the suitable amplitudes for the above matrix elements. The relevant renormalization constants, $Z_{V}$ and $Z_{A}$ have not been calculated yet. On the other hand, since the overlap action possesses exact chiral symmetry on the lattice, the ratio $Z_{V} / Z_{A}$ is expected to be close to 1 (it is exactly 1 for massless quarks). This allows us to get an estimate of the size of $f_{D_{s}^{*}}$ through the calculation of

$$
R\left(f_{D_{s}^{*}}, f_{D_{s}}\right)=\frac{Z_{A}}{Z_{V}} \frac{f_{D_{s}^{*}}}{f_{D_{s}}} \approx \frac{f_{D_{s}^{*}}}{f_{D_{s}}}
$$

since $f_{D_{s}}$ has been calculated very precisely in the literature. Our preliminary results for $R\left(f_{D_{s}^{*}}, f_{D_{s}}\right)=$ $1.15(10)$ on the lattices with $a=0.09 \mathrm{fm}$. The mixed action effects will be smaller for heavy-light mesons, and in the ratio its effects will be minimal.

\section{Conclusions}

In this work we reported preliminary results on the ground state hadron masses along with charm-strange meson decay constants by using a mixed action approach, comprising overlap va- 
lence quarks generated on the background of dynamical 2+1+1 flavours HISQ configurations. The results, in particular the hyperfine splitting of $1 \mathrm{~S}$ charmonia, are encouraging and suggest that the overlap valence on $2+1+1$ flavor HISQ configurations is a promising approach to do lattice QCD simulation with light, strange and charm quark together in the same lattice formulation. However, at these lattice spacings, the discretization errors of the overlap action for the charm, as evident from the dispersion relation, are not negligible, and are similar in size to those of the clover action.

This is a continuing study and we expect to be able to do suitable chiral and continuum extrapolations, to make experimentally relevant predictions for various charmed baryons. The splitting $\left(m_{\Omega_{c c c}}-\frac{3}{2} m_{J / \Psi}\right)$, between $J / \Psi$ and the unknown triply charmed baryon $\Omega_{c c c}$ was found to be $110_{-10}^{+20} \mathrm{MeV}$ and $120(10) \mathrm{MeV}$, on our lattices with $a=0.09$ and $0.06 \mathrm{fm}$ respectively. We are also studying heavy-light decay constants. Necessary renormalization constants will be calculated in future. Our preliminary results for $f_{D_{s}^{*}} / f_{D_{s}}$ (for which the renormalization constants approximately cancel) is 1.15(10), from our coarser lattice.

\section{Acknowledgement}

The computations were carried on the Blue Gene P of Indian Lattice Gauge Theory Initiative, Tata Institute of Fundamental Research (TIFR), Mumbai, and on the Konark cluster, NISER. We would like to thank A. Salve and K. Ghadiali for technical support. We are grateful to the MILC collaboration and in particular to S. Gottlieb, for providing us with the HISQ lattices. We also thank A. Li and A. Alexandru for help with numerical issues of overlap fermions.

\section{References}

[1] A. Bazavov et al. Phys. Rev. D 82, 074501 (2010); A. Bazavov et al., PoS(Lattice 2010)320 (2010); A. Bazavov et al. (MILC Collaboration), PoS(Lattice2012)158.

[2] E. Follana et al., Nucl. Phys. B (Proc. Suppl.) 129 and 130, 447 (2004); E. Follana et al., Phys. Rev. D75, 054502 (2007).

[3] H. Neuberger, Phys. Lett. B417 (1998) 141; ibid. B427 (19998) 353.

[4] M. Luscher, Phys. Lett. B428 (1998) 342.

[5] R. Edwards et al., Phys.Rev. D59 (1999) 094510.

[6] H. Fukaya et al., [JLQCD Collaboration], Phys. Rev. Lett. 98, 172001 (2007); Phys. Rev. D 77, 074503 (2008).

[7] A. Li et al. Phys. Rev. D82 (2010) 114501; N. Mathur et al., PoS LATTICE2010 (2010) 114.

[8] Y. Chen et al., Phys. Rev. D70 (2004) 034502, S.J. Dong et. al, Phys. Rev. Lett. 85 (2000) 5051-5054, ibid. Phys.Rev. D65 (2002) 054507.

[9] C.T.H. Davies et al., Phys.Rev. D81 (2010) 034506.

[10] S. Tamhankar et al., Phys. Lett. B638 (2006).

[11] T. Burch et al., Phys.Rev. D81 (2010) 034508; G.C. Donald et al., Phys.Rev. D86 (2012) 094501.

[12] C. Davies, et al., Phys.Rev. D82, 114504 (2010).

[13] N. Mathur et al., Phys.Rev. D66 (2002) 014502; R. Briceno et al., Phys.Rev. D86 (2012) 094504.

[14] O. Bar et al., Phys. Rev. D 67, 114505 (2003), ibid. 70, 034508 (2004); J.W. Chen et al., Phys. Rev. D75. 054501 (2007); K. Orginos et al., Phys. Rev. D77, 094505 (2008) and references therein.

[15] M. Lujan et al., Phys.Rev. D86 (2012) 014501. 